Corrigendum

\title{
Corrigendum to "Efficacy and Safety of Sipjeondaebo-Tang for Anorexia in Patients with Cancer: A Pilot, Randomized, Double-Blind, Placebo-Controlled Trial"
}

\author{
Chunhoo Cheon $\left(\mathbb{D},{ }^{1}\right.$ Jeong-Eun Yoo, ${ }^{2}$ Hwa-Seung Yoo $(D),{ }^{2}$ Chong-Kwan Cho, ${ }^{2}$ \\ Sohyeon Kang, ${ }^{1}$ Mia Kim, ${ }^{3}$ Bo-Hyoung Jang $₫{ }^{1},{ }^{1}$ Yong-Cheol Shin, ${ }^{1}$ and Seong-Gyu Ko $₫{ }^{1}$ \\ ${ }^{1}$ Department of Preventive Medicine, Korean Medical College, Kyung Hee University, Seoul, Republic of Korea \\ ${ }^{2}$ Dunsan Korean Medicine Hospital of Daejeon University, Daejeon, Republic of Korea \\ ${ }^{3}$ Department of Cardiovascular and Neurologic Disease (Stroke Center), College of Korean Medicine, Kyung Hee University, \\ Seoul, Republic of Korea \\ Correspondence should be addressed to Seong-Gyu Ko; epiko@khu.ac.kr
}

Received 16 May 2018; Accepted 21 June 2018; Published 30 July 2018

Copyright (C) 2018 Chunhoo Cheon et al. This is an open access article distributed under the Creative Commons Attribution License, which permits unrestricted use, distribution, and reproduction in any medium, provided the original work is properly cited.

In the article titled "Efficacy and Safety of Sipjeondaebo-Tang for Anorexia in Patients with Cancer: A Pilot, Randomized, Double-Blind, Placebo-Controlled Trial" [1], there was an error in the conclusion of the Abstract where the text reading "Sipjeondaebo-tang appears to have potential benefit for anorexia management in patients with cancer. Further largescale studies are needed to ensure the efficacy" should be corrected to "In the present study, Sipjeondaebo-tang did not show a significant effect on anorexia in patients with cancer. Further large-scale studies which compensate for the limitations of this study are needed to assess the efficacy".

This error in the reporting was brought to the attention of the journal by Professor Edzard Ernst, Emeritus Professor of Complementary Medicine, University of Exeter. The journal and the authors apologize for this misreporting of the conclusions.

\section{References}

[1] C. Cheon, J. Yoo, H. Yoo et al., "Efficacy and Safety of Sipjeondaebo-Tang for Anorexia in Patients with Cancer: A Pilot, Randomized, Double-Blind, Placebo-Controlled Trial," Evidence-Based Complementary and Alternative Medicine, vol. 2017, Article ID 8780325, 9 pages, 2017. 


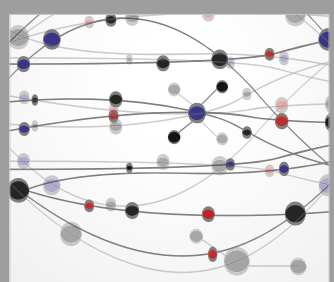

The Scientific World Journal
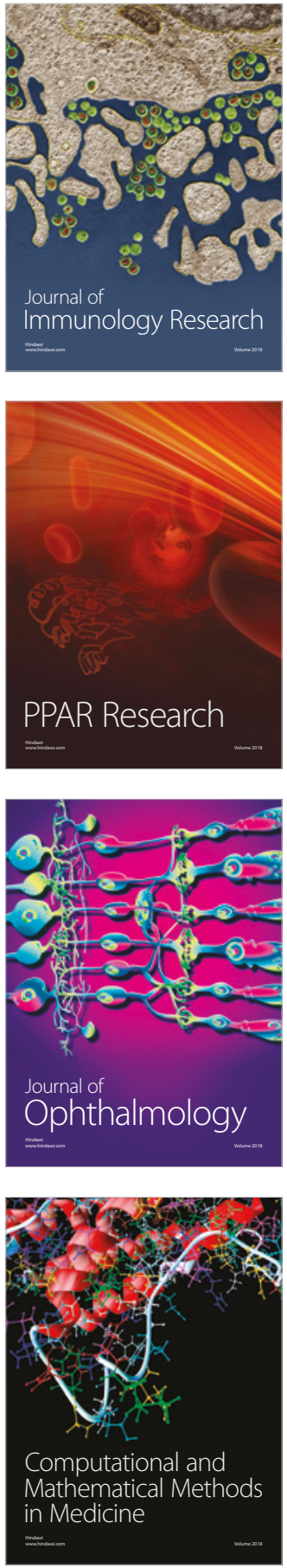

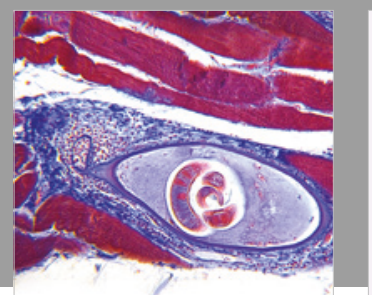

Gastroenterology Research and Practice

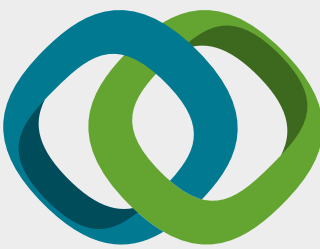

\section{Hindawi}

Submit your manuscripts at

www.hindawi.com
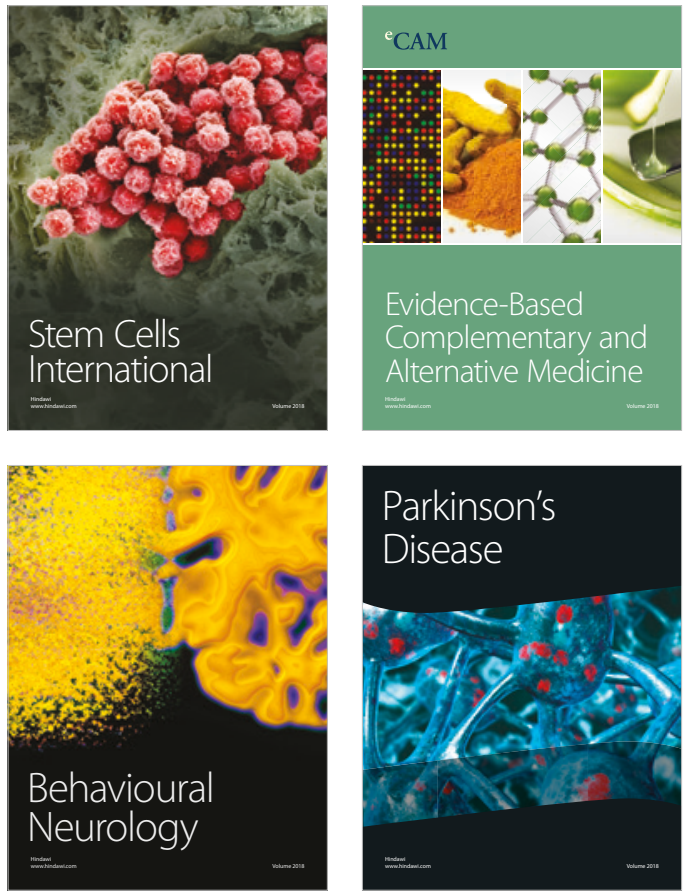

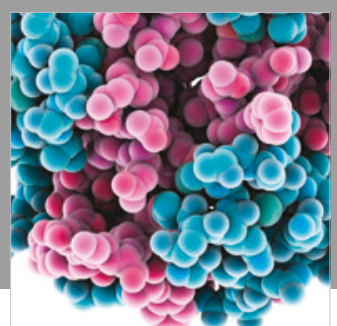

ournal of

Diabetes Research

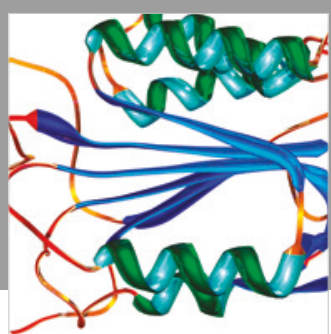

Disease Markers
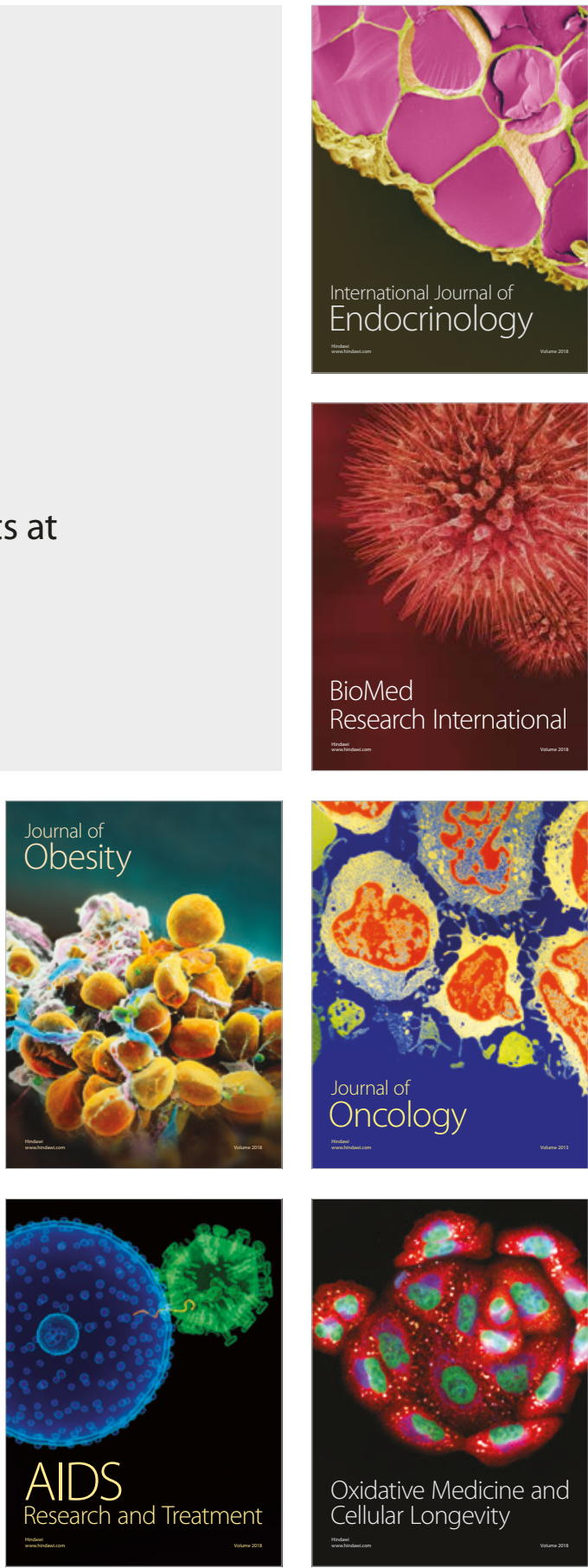